\title{
Roman and inverse Roman domination in graphs
}

\author{
Zulfiqar Zaman, M. Kamal Kumar and Saad Salman Ahmad \\ Department of Mathematics, Higher College of Technology \\ Muscat, Oman \\ e-mails: zulfiqarzaman7@gmail.com, \\ kamalmvz@gmail.com, saadretaj@hct.edu.om
}

Received: 5 February 2018 Revised: 16 September 2018 Accepted: 29 September 2018

\begin{abstract}
Motivated by the article in Scientific American [8], Michael A Henning and Stephen T. Hedetniemi explored the strategy of defending the Roman Empire. Cockayne defined Roman dominating function (RDF) on a Graph $G=(V, E)$ to be a function $f: V \rightarrow\{0,1,2\}$ satisfying the condition that every vertex $u$ for which $f(u)=0$. is adjacent to at least one vertex $v$ for which $f(v)=2$. For a real valued function $f: V \rightarrow R$ the weight of $f$ is $w(f)=\sum_{v \in V} f(v)$. The Roman Domination Number (RDN) denoted by $\gamma_{R}(G)$ is the minimum weight among all RDF in $G$. If $V-D$ contains a Roman dominating function $f^{1}: V \rightarrow\{0,1,2\}$, where $D$ is the set of vertices $v$ for which $f(v)>0$. Then $f^{1}$ is called inverse Roman dominating function (IRDF) on a graph $G$ w.r.t. $f$. The inverse Roman domination number (IRDN) denoted by $\gamma_{R}^{1}(G)$ is the minimum weight among all IRDF in $G$. In this paper we find few results of RDN and IRDN.
\end{abstract} Keywords: Domination number, Inverse domination number, Roman domination number.

2010 Mathematics Subject Classification: 05C69.

\section{Introduction}

The theory of domination was introduced by Claude Berge in 1958 [1] and Ore in 1962 [6]. The inspiration for this concept was drawn from the classical problem of covering a chessboard with a minimal number of chess pieces. The concept of domination theory in graphs is receiving much attention in the literature with more than hundred types of dominations being defined in the literature. One among those hundred is the Roman domination. Constantine the Great ruled the Roman Empire between 306 AD and 337 AD. Due to various conflicts with the neighboring territories, the Roman Empire was under severe attack. Due to this circumstance, the resources of the Roman Empire were completely declined. Emperor Constantine had to decide where to 
station his four field legions to protect eight regions. This challenge was overcome by placing the legions so that every region was either secured by its own legion or was securable by a neighbor with two legions, one of which could be sent to the undefended regions directly in case of conflict break out.

Ian Stewart in his article [8] argued that if Constantine had been a better mathematician, then the Roman Empire might have lasted a little longer than it did. The better way of deployment of legions was studied by C. S. ReVelle and K. E. Rosing through a form of zeroone integer programming [7]. Motivated by the article written by Ian Stewart, Michael A. Henning and Stephen T. Hedetniemi explored the strategy of defending the Roman Empire. They believed that the Roman Empire had the potential of saving the Emperor Constantine the Great, curtailing costs of maintaining legions while still defending the Roman Empire [3]. The deployment of legions is not the only problem associated with Roman domination. The same sort of mathematics is useful when one needs to decide the optimal location in the town for setting up a new fire station, a police station, hospitals, restaurants, etc. Many such optimization problems could be modeled using the concept of Roman domination.

Let $G(V, E)$ be a graph, A subset $S \subseteq V$ is a domination set of $G$, if for any vertex $u \in V-S$ there exist a vertex $v \in S$ such that $u v \in E$. The domination number of $G$, denoted by $\gamma(G)$ equals the minimum cardinality of the domination set.

A Roman dominating function (RDF) on a graph $G=(V, E)$ is defined as a function $f: V \rightarrow\{0,1,2\}$ satisfying the condition that every vertex $u$ for which $f(u)=0$ is adjacent to at least one vertex $v$ for which $f(v)=2$. For a real-valued function $f: V \rightarrow R$ the weight of $f$ is $w(f)=\sum_{v \in V} f(v)$. The Roman domination number (RDN) denoted by $\gamma^{1}(G)$ is the minimum weight among all RDF in $G$.

The positions of legions are kept secret in order to take advantage over their enemy's strategies. But if the enemies come to know the positions of the legions, then the best chance of attack is in the places where no legions are placed, so that the time lags in moving the legions from the adjacent regions can be taken as an advantage. Hence, if the emperor comes to know that the enemies know their legions positions. In order to surprise the enemies and create great damage, the question of optimal reorganizing of the legions still defending the Roman Empire need to be answered. Hence we define the inverse Roman dominating function.

An Inverse Roman Dominating Function (IRDF) is also a Roman dominating function. If $V-D$ contains a Roman dominating function $f^{1}: V \rightarrow\{0,1,2\}$, where $D$ is the set of vertices $v$ for which $f(v)>0$, then $f^{1}$ is called Inverse Roman Dominating Function (IRDF) on a graph $G$, with respect to a Roman dominating function $f$. The inverse Roman Domination Number (IRDN) denoted by $\gamma_{R}^{1}(G)$ is the minimum weight among all IRDF in $G$. For any undefined terms or notation in this paper, we refer Harary [2].

\section{Preliminary results $[4,5]$}

Proposition 2.1. For any given graph $G(n, m), \gamma_{R}(G) \leq \gamma^{1}(G)$.

Corollary 2.1. $\gamma_{R}(G) \leq \gamma_{R}^{1}(G) \leq n$. 
Proposition 2.2. For any complete graph $K_{n}$, with $n$ vertices, $\gamma_{R}\left(K_{n}\right)=\gamma_{R}^{1}\left(K_{n}\right)=2$.

Proposition 2.3. For the classes of cycle $C_{n}$ with $n$ vertices, $\gamma_{R}(G)=\gamma_{R}^{1}(G)=\left\lceil\frac{2 n}{3}\right\rceil$.

Proposition 2.4. For the classes of paths $P_{n}$ with $n>2$ vertices,

$$
\gamma_{R}^{1}(G)=\left\{\begin{array}{cc}
\left\lceil\frac{2 n}{3}\right\rceil+1 & \text { if } n \equiv 0(\bmod 3) \\
\left\lceil\frac{2 n}{3}\right] & \text { Otherwise }
\end{array}\right\} .
$$

Proposition 2.5. For the classes of wheel $W_{n}$ with $n \geq 4$ vertices, $\gamma_{R}^{1}(G)=\left\lceil\frac{2(n-1)}{3}\right\rceil$.

Proposition 2.6. For the classes of star $K_{1, n-1}$ with $n$ vertices, $\gamma_{R}^{1}(G)=n$.

Proposition 2.7. For the classes of ladder $G_{2, n}, \gamma_{R}^{1}(G)=n+1$.

Proposition 2.8. For any graph $G$ of order $n$ with maximum degree $\Delta$ and minimum degree $\delta$,

$$
\left\lceil\frac{2 n}{\Delta+1}\right\rceil \leq \gamma_{R}^{1}(G) \leq n-\delta+1 \text {. }
$$

\section{Definitions}

Banana Graph: An $(n, k)$-banana tree is a graph obtained by connecting one leaf to each of $n$ copies of a $k$-star graph with a single root vertex that is distinct from all the stars, denoted by $B(n, k)$.

Lollipop Graph: An $(m, n)$-lollipop graph is the graph obtained by joining a complete graph $K_{m}$ to path $P_{n}$ with a bridge, denoted by $L(m, n)$.

Book Graph: The $m$ book graph is defined as the graph Cartesian product $S_{m+1} \times P_{2}$, where $S_{m}$ is a star graph and $P_{2}$ is the path graph, denoted by $B_{m}$.

Stacked Book Graph: The $(m, n)$-stacked book graph is defined as the graph Cartesian product $S_{m+1} \times P_{n}$, where $S_{m}$ is a star graph and $P_{n}$ is the path graph, denoted by $B_{m, n}$. Therefore the graph corresponding to the edges of $n$ copies of an $m$-page "book" stacked one on top of another and is a generalization of the book graph.

Crown Graph: A crown graph on $2 n$ vertices is a graph with two sets of vertices $\left\{u_{1}, u_{2}, \ldots, u_{n}\right\}$ and $\left\{v_{1}, v_{2}, \ldots, v_{n}\right\}$ with an edge from $u_{i}$ to $v_{j}$ whenever $i \neq j$, denoted by $S_{n}^{0}$.

Bipartite Graph: A graph is said to be bipartite if its vertex set can be partitioned into two disjoint subsets $V=v_{1} \cup v_{2}$, such that every edge has the form $e=(a, b)$, where $a \in v_{1}$ and $b \in v_{2}$ also no vertices both in $v_{1}$ or both in $v_{2}$ are adjacent.

Complete Bipartite Graph: A complete bipartite graph $K_{m, n}$ is a bipartite graph that has each vertex from one set adjacent to each vertex of another set and no two graph vertices within the same set are adjacent. 


\section{Main results}

Theorem 4.1. Let $G=B_{m, n}$. Then, $\gamma_{R}\left(B_{m, n}\right)=2 n+1$ and $\gamma_{R}^{1}\left(B_{m, n}\right)=n m$.

Proof: Let $v \in V$ with $\operatorname{deg}(v)=n, \operatorname{deg}(v)=2, \operatorname{deg}(v)=m-1$ and $\operatorname{deg}(v)=1$ are labeled as $r_{1}, v_{i}$, $1 \leq i \leq n, w_{i}, 1 \leq i \leq n$ and $u_{i, j}, 1 \leq i \leq n, 1 \leq j \leq m-2$, respectively. Let $f$ be a $\gamma_{R}$-function with $f=\left(V_{0}, V_{1}, V_{2}\right)$, by definition each $v \in V_{0}$ will be adjacent to at least one vertex $u \in V_{2}$. Without loss of generality, the maximum degree in $G$ is $\Delta=m-1$. Hence, $f\left(w_{i}\right)=2,1 \leq i \leq n$, then by definition $N\left(w_{i}\right)=0,1 \leq i \leq n$, i.e., $f\left(v_{i}\right)=f\left(u_{i j}\right)=0, \forall i, j$ and in $G$ with $\left|V_{1}\right|=1, f\left(r_{1}\right)=1$. Therefore, $\gamma_{R}\left(B_{n, m}\right)=2(n)+1=2 n+1$. Let $f^{1}$ be the inverse Roman dominating function with $f^{1}\left(v_{i}\right)=2,1 \leq i \leq n$. As $w_{i} u_{i} r_{1}, 1 \leq i \leq n$ forms a path $P_{3}$ in which $f\left(w_{i}\right)=2,1 \leq i \leq n$ and $f\left(r_{1}\right)=1$. Therefore, $f^{1}\left(u_{i j}\right)=1,1 \leq i \leq n, 1 \leq j \leq(m-2)$. Hence $\gamma_{R}^{1}\left(B_{m, n}\right)=2(n)+n(m-2)$ $=n m$.

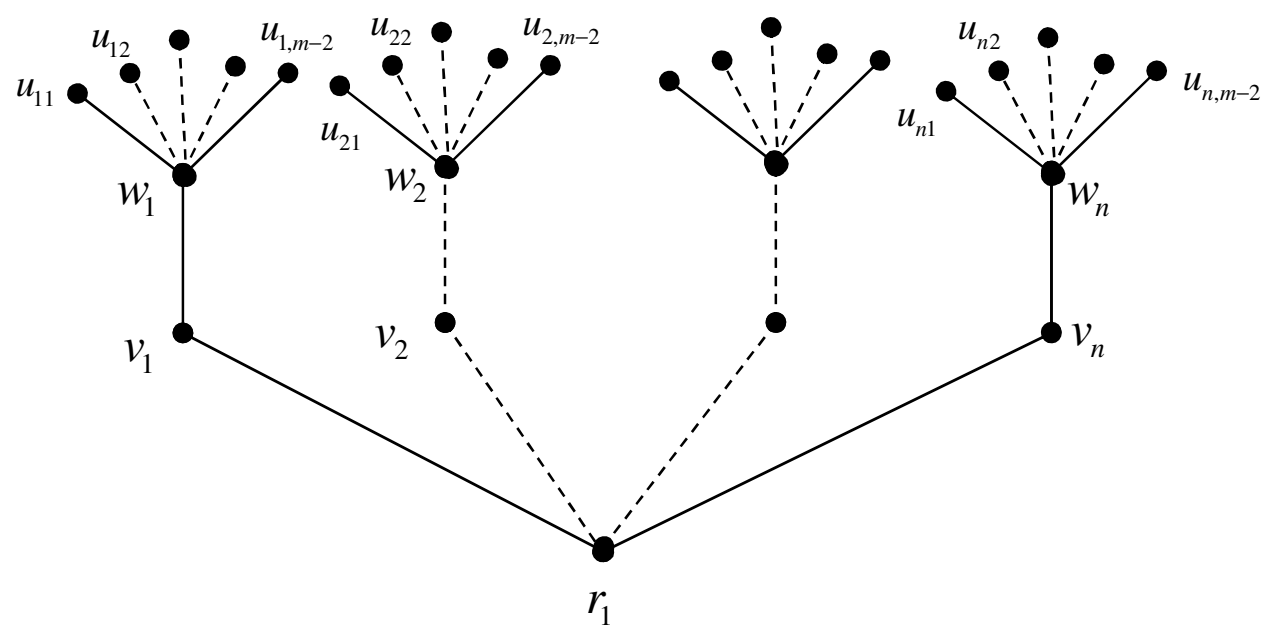

Figure 1. Banana tree $B_{n, m}$.

Theorem 4.2. Let $G=L(m, n)$. Then,

$$
\gamma_{R}(L(m, n))=\left\{\begin{array}{c}
\left\lceil\frac{2 n}{3}\right\rceil+2 \text { if } n \equiv 0(\bmod 3) \\
\left\lceil\frac{2(n-1)}{3}\right\rceil+2 \quad \text { Otherwise }
\end{array}\right\}
$$

and

$$
\gamma_{R}^{1}(L(m, n))=\left\{\begin{array}{cc}
\left\lceil\frac{2(n-1)}{3}\right\rceil+2 & \text { if } n \equiv 0(\bmod 3) \\
\left\lceil\frac{2 n}{3}\right\rceil+2 & \text { Otherwise }
\end{array}\right\} .
$$

Proof: Let $P_{n}$ be a path with $u_{i}, 1 \leq i \leq n$ vertices connected to a complete graph $K_{m}$ with $v_{i}$, $1 \leq i \leq n$ vertices along a bridge $v_{1} u_{1}$ and $f\left(v_{1}\right)=2$. 


\section{Consider two cases.}

Case 1: If $n \equiv 0(\bmod 3)$, there exists a unique minimal Roman domination set

$$
D=\left\{u_{3 p} / 1 \leq p \leq \frac{n}{3}\right\}
$$

with $f\left(u_{i}\right)=2, u_{i} \in D$. Hence $\gamma_{R}(L(m, n))=2+\left\lceil\frac{2 n}{3}\right\rceil$. For the inverse Roman dominating function $f^{1}$, let $f^{1}\left(v_{i}\right)=2$, for $i \neq 1$. Then, there exist an unique inverse Roman domination set $D^{1}$ given by $D^{1}=\left\{u_{i-1} / u_{i} \in D\right\}$, with $f^{1}\left(u_{i}\right)=2, u_{i} \in D^{1}$, therefore

$$
\gamma_{R}^{1}(L(m, n))=2+\left\lceil\frac{2(n-1)}{3}\right\rceil .
$$

Case 2: If $n \not \equiv 0(\bmod 3)$, there exists a minimal Roman dominating function with $f\left(u_{n}\right)=1$, for $n=3 p-1$ and $f\left(u_{n}\right)=0$ for $n=3 p+1, p \in N$. The Roman domination set is given by

$$
D=\left\{u_{3 p} / 1 \leq p \leq \frac{n}{3}\right\} \cup\left\{u_{n}\right\} \text { for } n=3 p-1
$$

or

$$
D=\left\{u_{3 p} / 1 \leq p \leq \frac{n}{3}\right\} \text { for } n=3 p+1 .
$$

Hence $\gamma_{R}(L(m, n))=2+\left\lceil\frac{2(n-1)}{3}\right\rceil$. For the inverse Roman domination function $f^{1}, f^{1}\left(v_{i}\right)=2$, $i \neq 1$. Then the inverse Roman domination set given by $D^{1}=\left\{u_{i-1} / u_{i} \in D\right\}$ with $f^{1}\left(u_{i}\right)=2$, $u_{i} \in D^{1}$, therefore $\gamma_{R}^{1}(L(m, n))=\left\lceil\frac{2 n}{3}\right\rceil+2$.

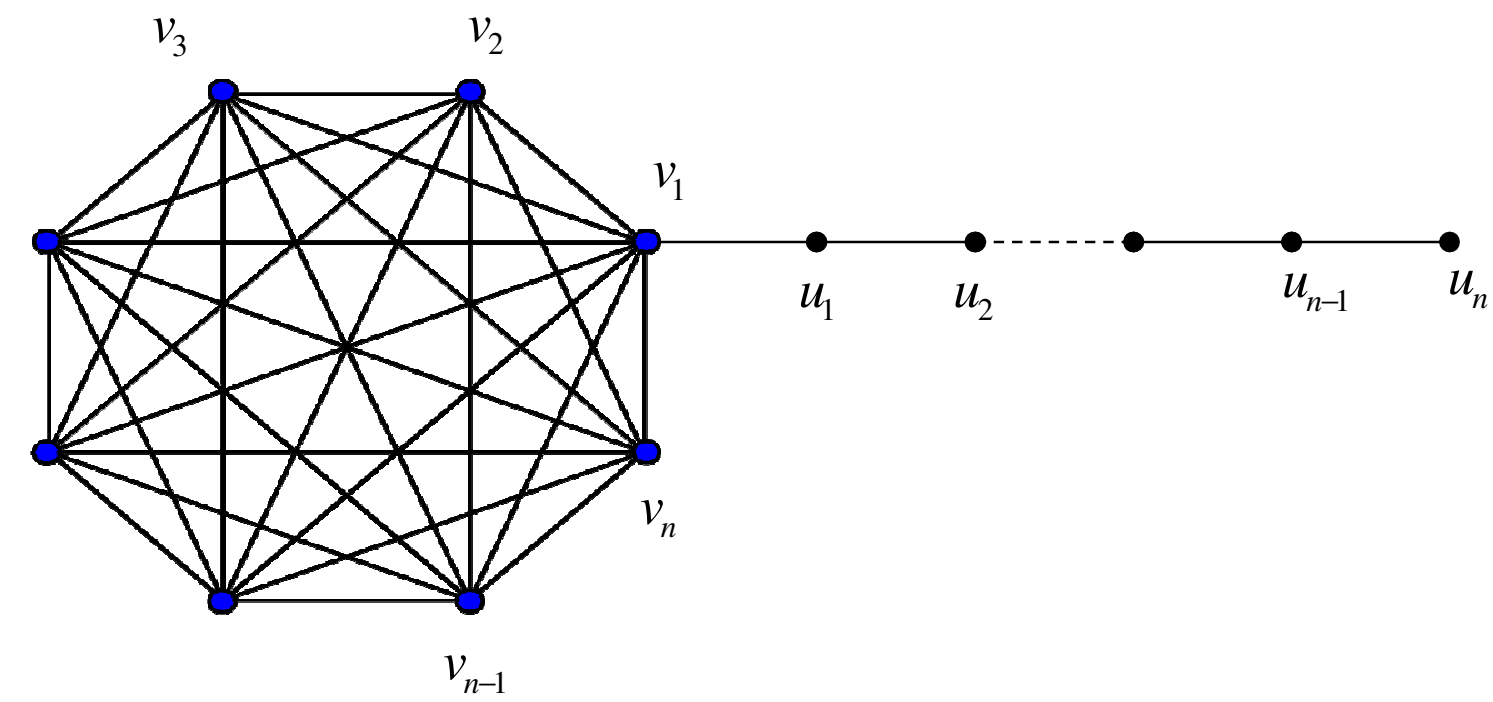

Figure 2. Lollipop graph $L(m, n)$. 
Theorem 4.3. Let $G=B_{n}$. Then, $\gamma_{R}\left(B_{n}\right)=\gamma_{R}^{1}\left(B_{n}\right)=4$.

Proof: The graph $G=B_{n}$ is Cartesian product of $S_{n+1}$ and $P_{2}$. Let $f$ be a $\gamma_{R}$-function with $f=\left(V_{0}, V_{1}, V_{2}\right)$, by definition each $v \in V_{0}$ will be adjacent to at least one vertex $u \in V_{2}$. In $B_{n}$, each star has one vertex with $\operatorname{deg}(v)=n,|\Delta(G)|=2$ hence $\left|V_{2}\right|=2,\left|V_{1}\right|=0$. Therefore $\gamma_{R}\left(B_{n}\right)=2+2=4$. For the inverse Roman dominating function $f^{1}$ there are $n$ sets of $P_{2}$. Hence $\left|V_{2}\right|=n,\left|V_{1}\right|=0$. Therefore, $\gamma_{R}^{1}\left(B_{n}\right)=2 n$.

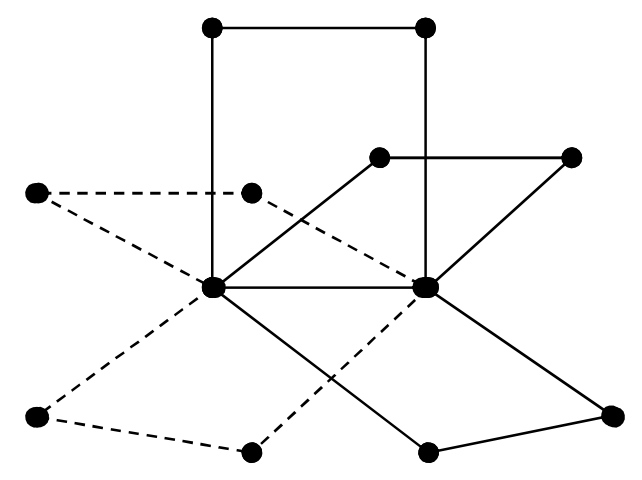

Figure 3: Book graph $B_{n}$.

Theorem 4.4. Let $G=S_{n}^{0}$. Then, $\gamma_{R}\left(S_{n}^{0}\right)=\gamma_{R}^{1}\left(S_{n}^{0}\right)=4$.

Proof: Let $G=S_{n}^{0}$ consist of two disjoint sets of vertices $U=\left\{u_{i}\right\}, 1 \leq i \leq n$ and $V=\left\{v_{j}\right\}$, $1 \leq j \leq n$, with an edge from $u_{i}$ to $v_{j}$ whenever $i \neq j, 1 \leq i, j \leq n$. Hence for Roman dominating function $f$, we have $f\left(u_{i}\right)=f\left(v_{j}\right)=2, i=j$, for exactly one value of $i$ or $j, 1 \leq i, j \leq n$. Hence $\gamma_{R}\left(S_{n}^{0}\right)=2+2=4$. For inverse Roman dominating function $f^{1}$ due to symmetry of graph $f^{1}\left(u_{i}\right)=f^{1}\left(v_{j}\right)=2, i=j$, for any exactly value of $i$ or $j, 1 \leq i, j \leq n$ such that $\left\{u_{i}, v_{j}\right\} \notin D$. Hence $\gamma_{R}^{1}\left(S_{n}^{0}\right)=2+2=4$.

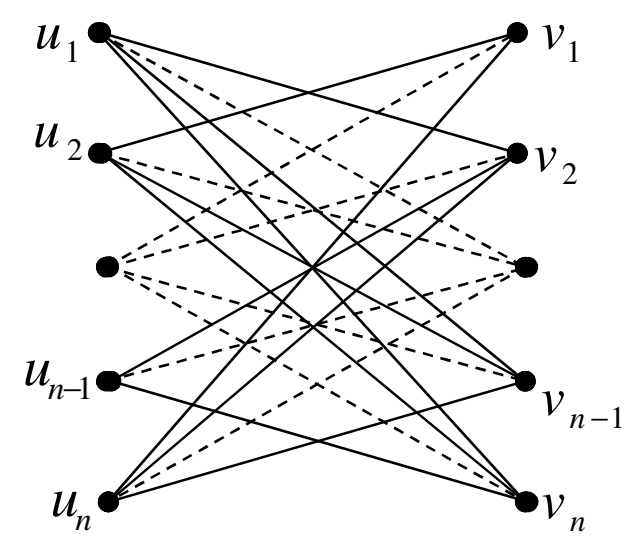

Figure 4. Crown graph $S_{n}^{0}$. 
Theorem 4.5: Let $G=K_{m, n}$. Then, $\gamma_{R}\left(K_{m, n}\right)=\gamma_{R}^{1}\left(K_{m, n}\right)=4$.

Proof: The vertex set of the complete bipartite graph can be partitioned into two sets $U=\left\{u_{i}\right\}$, $1 \leq i \leq m$ and $V=\left\{v_{j}\right\}, 1 \leq j \leq n$, such that each vertex from one set is adjacent to each vertex of another set and no two graph vertices within the same set are adjacent. For Roman dominating function $f, f\left(u_{i}\right)=2,1 \leq i \leq m$ and $f\left(v_{j}\right)=2,1 \leq j \leq n$. Therefore, $\gamma_{R}\left(K_{m, n}\right)=2+2=4$. For the inverse Roman dominating function $f^{1}$ due to symmetry of graph, $f^{1}\left(u_{i}\right)=2,1 \leq i \leq m$ and $f^{1}\left(v_{j}\right)=2,1 \leq j \leq n$, such that $\left\{u_{i}, v_{j}\right\} \notin D$. Hence $\gamma_{R}^{1}\left(K_{m, n}\right)=2+2=4$.

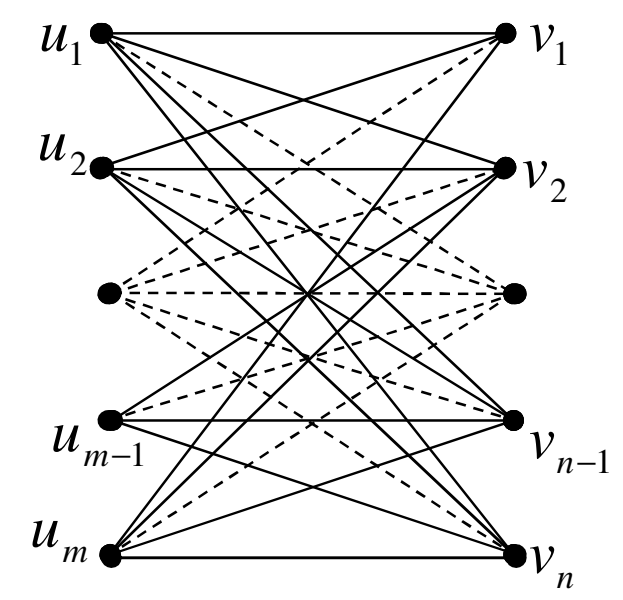

Figure 5. Complete bipartite graph $K_{m, n}$.

Theorem 4.6: Let $G=K_{m_{1}, m_{2}, \ldots, m_{n}}, m_{1} \leq m_{2} \leq \ldots \leq m_{n}$.

a) If $m_{1}=1$, then $\gamma_{R}(G)=2$ and $\gamma_{R}^{1}(G)=\left\{\begin{array}{lll}2 & \text { for } & m_{2}=1 \\ 3 & \text { for } & m_{2}=2 \\ 4 & \text { for } & m_{2}>2\end{array}\right.$

b) If $m_{1}=2$, then $\gamma_{R}(G)=3$ and $\gamma_{R}^{1}(G)=\left\{\begin{array}{lll}3 & \text { for } & m_{2}=2 \\ 4 & \text { for } & m_{2}>2\end{array}\right.$

c) If $m_{1} \geq 3$, then $\gamma_{R}(G)=\gamma_{R}^{1}(G)=4$.

Proof: The vertex set of the complete $n$ partite graph can be partitioned into $n$ sets $U_{1}=\left\{u_{11}, u_{12}, \ldots u_{1 m_{1}}\right\}, U_{2}=\left\{u_{21}, u_{22}, \ldots u_{2 m_{2}}\right\}, \ldots, U_{n}=\left\{u_{n 1}, u_{n 2}, \ldots u_{n m_{n}}\right\}$ such that each vertex from one set is adjacent to each vertex of another set and no two graph vertices within the same set are adjacent. The following cases are obtained to find RDF and IRDF.

Case 1: If $m_{1}=m_{2}=1$, then there exists a unique Roman and inverse Roman domination set such that $|D|=\left|D^{1}\right|=1$. Without loss of generality, let $f\left(u_{11}\right)=f^{1}\left(u_{21}\right)=2$, therefore $\gamma_{R}(G)=\gamma_{R}^{1}(G)=2$. If $m_{1}=1, m_{2}=2$, then $f$ be a $\gamma_{R}$-function with $f\left(V_{0}, V_{1}, V_{2}\right)$ given $\left|V_{0}\right|=$ $\left|V_{1}\right|=0,\left|V_{2}\right|=1$. Without loss of generality, let $f\left(u_{11}\right)=2$, therefore $\gamma_{R}(G)=2$. For IRDF let $f^{1}$ be $\gamma_{R}^{1}$ function with $f^{1}\left(V_{0}^{1}, V_{1}^{1}, V_{2}^{1}\right)$ given $\left|V_{0}^{1}\right|=0,\left|V_{1}^{1}\right|=1,\left|V_{2}^{1}\right|=1$. Without loss of 
generality, let $f^{1}\left(u_{21}\right)=2, f^{1}\left(u_{22}\right)=1$. Therefore $\gamma_{R}^{1}(G)=3$. Similarly, if $m_{1}=1, m_{2}>2$, then $\gamma_{R}(G)=2$. i.e., $f\left(u_{11}\right)=2$. For IRDF $f^{1}\left(u_{21}\right)=2, f^{1}\left(u_{31}\right)=2$. Therefore $\gamma_{R}^{1}(G)=4$. Hence the result.

Case 2: Similar to Case 1, if $m_{1}=m_{2}=2$, then $\gamma_{R}(G)=\gamma_{R}^{1}(G)=3$, i.e., $f\left(u_{11}\right)=2, f\left(u_{12}\right)=1, f^{1}\left(u_{21}\right)=2$, $f^{1}\left(u_{22}\right)=1$. If $m_{1}=2, m_{2} \geq 3$, then $\gamma_{R}(G)=3$, i.e., $f\left(u_{11}\right)=2, f\left(u_{12}\right)=1, f^{1}\left(u_{21}\right), f^{1}\left(u_{31}\right)=2$. Therefore $\gamma_{R}^{1}(G)=4$. Hence the result.

Case 3: Similar to Case 1, if $m_{1} \geq 3$, then $\gamma_{R}(G)=\gamma_{R}^{1}(G)=4$, i.e., $f\left(u_{11}\right)=f\left(u_{21}\right)=2$, and $f^{1}\left(u_{12}\right)=f^{1}\left(u_{22}\right)=2$. Hence the result.

Theorem 4.7: Let $G=B(m, n)$. Then, $\gamma_{R}(B(m, n))=2 n$ and $\gamma_{R}^{1}(B(m, n))=2(m+n)-4$.

Proof: The graph $B(m, n)$ consists of a star graph $S_{m+1}$ and a path graph $P_{n}$. For a minimal Roman dominating function $f$, each star has one vertex with $\operatorname{deg}(v)=m, \mid \Delta(G)=n$ hence $\left|V_{2}\right|=n,\left|V_{1}\right|=0$. Therefore, $\gamma_{R}(B(m, n))=2 n$. For the inverse Roman dominating function $f^{1}$ we have pendant vertices of the star graph, therefore, $f^{1}\left(u_{i}\right)=2,1 \leq i \leq(m-1)$ and $f^{1}\left(v_{j}\right)=2$, $1 \leq j \leq(n-1)$. Hence, $\gamma_{R}^{1}(B(m, n))=(m-1) 2+2(n-1)=2(m+n)-4$.

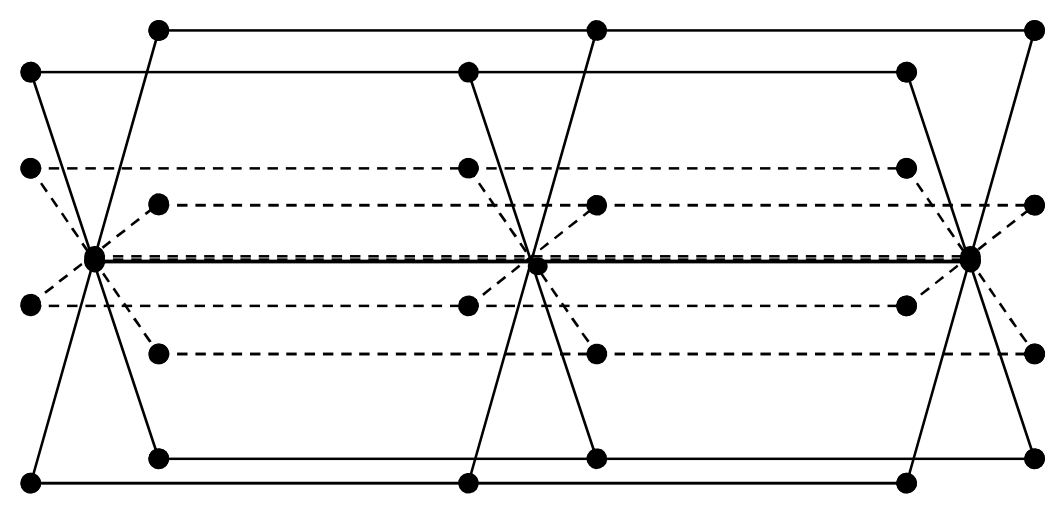

Figure 6. Stacked book graph $B(m, n)$.

Proportion 4.8: For a graph $G$ on $n$ vertices, $\gamma(G)=\gamma_{R}(G) \underline{\text { iff }} G=\overline{K_{n}}$, and $\gamma_{R}^{1}(G)$ does not exist.

Proof: Let $G=\overline{K_{n}}$, then the vertex set in $G$ can be partitioned into $V_{0}, V_{1}, V_{2}$ that indicates the vertices of $G$ for which $f(u)=0, \forall u \in V_{0}, f(v)=1, \forall v \in V_{1}, f(w)=2, \forall w \in V_{2}$ respectively. If $G=\overline{K_{n}}$, then $\left|V_{0}\right|=\left|V_{2}\right|=0$. Hence $\left|V_{1}\right|=|V|=n$. Therefore, there exist no vertices in $V-D$ for the inverse Roman dominating function $f^{1}$. Hence IRDF does not exist. 
Proportion 4.9: If $G$ is a graph on $n$ vertices with a vertex of degree $n-1$, then $\gamma(G)=1$, $\gamma_{R}(G)=2$ and $2 \leq \gamma_{R}^{1}(G) \leq n-\delta+1$.

Proof: The result follows from Proposition 2.8 [4].

Proportion 4.10: If $G$ is a graph on $n$ vertices, then $\gamma_{R}(G)=\gamma_{R}^{1}(G)$ iff $G$ has two vertices of degree $n-1$ or the graph $G$ is symmetric in nature.

Proof: Let the given graph is symmetric in nature then the vertex set of $G$ can be partitioned in two sets $V_{0}, V_{1}, V_{2}$ and $V_{0}^{1}, V_{1}^{1}, V_{2}^{1}$ for RDF and IRDF such that $\left|V_{0}\right|=\left|V_{0}^{1}\right|,\left|V_{1}\right|=\left|V_{1}^{1}\right|$, $\left|V_{2}\right|=\left|V_{2}^{1}\right|$. If there exist $u, v \in V$, such that $\operatorname{deg}(u)=\operatorname{deg}(v)=n-1$, then $f(u)=f^{1}(v)=2$. Hence the proof.

\section{Open problems}

Open Problem 1. Find the sufficient condition and characterize the graph for which $\gamma_{R}(G) \leq \gamma_{R}^{1}(G), \gamma_{R}(G)=\gamma_{R}^{1}(G) \& \gamma_{R}(G) \neq \gamma_{R}^{1}(G)$.

Open Problem 2. Find bounds of $\gamma_{R}^{1}(G)$ for various classes of graphs and also for arbitrary graph.

\section{References}

[1] Berge, C. (1958) Theory of Graphs and Its Applications, Methuen, London.

[2] Harary, F. (1975) Graph Theory, Addison Wiley, Reading Mass.

[3] Henning, M. A., \& Hedetniemi, S. T. (2003) Defending the Roman Empire - A new strategy, Discrete Mathematics, 266, 239-251.

[4] Kamal Kumar, M., \& Murali, R. (2014) Inverse Roman domination in some classes of graphs. International Journal of Computer Application, 4(4), 219-238.

[5] Kamal Kumar, M., \& Sudershan Reddy, L. (2013) Inverse Roman domination in graphs, Discrete Mathematics Algorithm and Application, 5(3), 1-4.

[6] Ore, O. (1962) Theory of Graphs. American Mathematical Society Colloquium Publications, 38 (American Mathematical Society, Providence, RI).

[7] ReVelle. C. S, Rosing. K. E. (2000) Defendens imperium Romanum: A classical problem in military, Strategy, Amer. Math. Monthly, 107(7), 585-594.

[8] Stewart, I. (1999) Defend the Roman Empire! Scientific American, 281(6), 136-139. 